PROCEEDINGS OF THE

AMERICAN MATHEMATICAL SOCIETY

Volume 127, Number 4, April 1999, Pages 1103-1108

S 0002-9939(99)04642-0

\title{
ANTICOMMUTING DERIVATIONS
}

\author{
STEEN PEDERSEN
}

(Communicated by Palle E. T. Jorgensen)

\begin{abstract}
We show that there are no non-trivial closable derivations of a $C^{*}$ algebra anticommuting with an ergodic action of a compact group, supposing that the set of squares is dense in the group. We also show that there are no non-trivial closable densely defined rank one derivations on any $C^{*}$-algebra.
\end{abstract}

\section{INTRODUCTION}

This paper is concerned with the theory of unbounded derivations on $C^{*}$-algebras, a subject whose study was motivated by questions in quantum physics and statistical mechanics. Derivations are also used in the study of other areas in mathematics, for example differentiation on manifolds, non-commutative geometry, and transformation groups. This paper was motivated in part by a study of representations of the Heisenberg Lie algebra. See, for example, [BR79], [JM84], [Sa91] for surveys of some of these developments.

Let $(\mathfrak{A}, G, \alpha)$ be a $C^{*}$-dynamical system and let $\delta$ be a (unbounded) derivation of $\mathfrak{A}$. That is, $\mathfrak{A}$ is a $C^{*}$-algebra, $G$ a topological group and $\alpha$ is a continuous homomorphism of $G$ into the group of $*$-automorphisms of $\mathfrak{A}$ equipped with the topology of pointwise convergence. There is an extensive literature (see $[\mathrm{Br} 86]$ and [Sa91] for surveys) showing that under a variety of conditions on $\alpha$ and/or $\delta$ commutation of $\alpha$ and $\delta$ have consequences for the structure of $\delta$. Versions of many of these results remain valid if we replace the commutation of $\alpha$ and $\delta$ by anticommutation (defined below). The purpose of this paper is to show that stronger conclusions are possible in the presence of the anticommutation relation, than are possible in the presence of the commutation relation. Specifically, we show that if $(\mathfrak{A}, G, \alpha)$ is a compact ergodic $C^{*}$-dynamical system, $\left\{g^{2}: g \in G\right\}$ is dense in $G$ and $\delta$ is a closable densely defined derivation, then anticommutation of $\alpha$ and $\delta$ implies $\delta=0$. The proof depends on the fact that there are no non-trivial densely defined rank one derivations of any $C^{*}$-algebra; we prove this below.

Background material on unbounded derivations, $C^{*}$-algebras, and group actions on $C^{*}$-algebras can, for example, be found in [BR79], [Br86], and [Sa91].

Let $R$ and $S$ be bounded everywhere defined operators on a Banach space $X$, and suppose $T$ is a densely defined (possibly unbounded) operator on $X$. By $R T \subset T S$ we mean that $S$ maps the domain of $T$ into itself and $R T x=T S x$ for any $x$ in the domain of $T$. Let $(\mathfrak{A}, G, \alpha)$ be a $C^{*}$-dynamical system and let $\delta$ be a (possibly

Received by the editors July 5, 1996 and, in revised form, July 25, 1997.

1991 Mathematics Subject Classification. Primary 46L57, 47B47; Secondary 46L55, 47D45.

Key words and phrases. Derivation, anticommutation, Heisenberg commutation.

(C)1999 American Mathematical Society 
unbounded) derivation of $\mathfrak{A}$. We say that $\alpha$ and $\delta$ commute when $\alpha_{g} \delta \subset \delta \alpha_{g}$ for all $g$ in $G$. We say that $\alpha$ and $\delta$ anticommute when $\alpha_{g^{-1}} \delta \subset \delta \alpha_{g}$ for all $g$ in $G$. Note that if $R S=-S R$, then a formal power series expansion yields $\exp (-t R) S=S \exp (t R)$ for all real numbers $t$. Hence, if $G$ is the additive group of real numbers, then anticommutation of $\alpha$ and $\delta$ corresponds to anticommutation, in the usual sense, of $\delta$ and the infinitesimal generator of $\alpha$.

Before proving the main results, we want to point out that formally an anticommuting pair $(\alpha, \delta)$ corresponds to a representation of the Heisenberg commutation relation. The following computations will be formal, in particular, we will ignore the domain problems associated with unbounded operators. Let $G$ be the additive group of real numbers and let $\gamma$ be the generator of $\alpha$. Suppose there exist operators $H$ and $K$ so that $\delta(a)=K a-a K$ and $\gamma(a)=H a-a H$, and let $C=H K-K H$. It is easy to see that (formally) $\alpha_{-t} \delta=\delta \alpha_{t}$ implies $\gamma \delta=-\delta \gamma$ which in turn implies $H C=C H$ and $K C=C K$, hence $H, K$ and $C$ is a representation of the Heisenberg Lie algebra, $C$ being the central element. Hence, our main result shows that certain representations of the Heisenberg commutation relations are impossible. It should be noted that commutation of $\alpha$ and $\delta$ corresponds to $H K-K H$ being a scalar multiple of the identity.

\section{RANK ONE DERIVATIONS}

The main result in this section is that $C^{*}$-algebras do not admit non-trivial closed rank one $*$-derivations. This is first proved for a densely defined derivation whose range is a subset of its domain. This is relatively easy, but it illustrates one aspect of the proof of the main result and it is used in the proof of the main result.

Recall, a derivation of a $C^{*}$-algebra is $\mathfrak{A}$ is a linear map $\delta$ from a subalgebra $\mathcal{D}(\delta)$, the domain of $\delta$, into $\mathfrak{A}$ that satisfies Leibnitz's rule:

$$
\delta(a b)=\delta(a) b+a \delta(b)
$$

for all $a$ and $b$ in $\mathfrak{A}$. A $*$-derivation is a derivation $\delta$ whose domain is a $*$-subalgebra of $\mathfrak{A}$ with the property that

$$
\delta\left(a^{*}\right)=\delta(a)^{*}
$$

for all $a$ in $\mathfrak{A}$.

Proposition 2.1. Let $\delta$ be a densely defined derivation on a $C^{*}$-algebra, whose range is a subset of its domain. If the rank of $\delta$ is at most one, then $\delta=0$.

Proof. Let $\delta$ be a derivation of a $C^{*}$-algebra $\mathfrak{A}$. Suppose $\delta(\mathcal{D}(\delta)) \subset D(\delta)$ and the range of $\delta$ is one dimensional; then

$$
\delta(a)=h(a) R,
$$

for any $a$ in $\mathfrak{A}$, where $h$ is a function from $\mathcal{D}(\delta)$ into the complex numbers and $R$ is a non-zero element in $\mathfrak{A}$. Note, $R$ is in the domain of $\delta$, because $\delta(\mathcal{D}(\delta)) \subset D(\delta)$. Fix $b$ in $\mathcal{D}(\delta)$ with $\delta(b)=R$, i.e., $h(b)=1$. First we will show

$$
R a+a R \text { is a scalar multiple of } R
$$

for any $a$ in $\mathfrak{A}$. By density of $\mathcal{D}(\delta)$ in $\mathfrak{A}$ it is enough to show this for $a$ in $\mathcal{D}(\delta)$. Let $a$ be in $\mathcal{D}(\delta)$. If $h(a) \neq 0$, then $h(a)(a R+R a)=\delta\left(a^{2}\right)=h\left(a^{2}\right) R$. If $h(a)=0$, then $h(a b+b a) R=\delta(a b+b a)=a R+R a$. This proves (2.1). Next we will show that

$$
R^{2}=0 .
$$


If $h(R)=0$, then expanding $\delta\left(b^{2}\right)$ we get $h\left(b^{2}\right) R=R b+b R$. Applying $\delta$ to this equality and using $\delta(R)=0$ yields $0=2 R^{2}$. Next suppose $h(R) \neq 0$. First note that applying $\delta$ to $R^{2}$ we get

$$
h\left(R^{2}\right) R=\delta\left(R^{2}\right)=2 h(R) R^{2},
$$

so that

$$
R^{2}=k R,
$$

where $k=h\left(R^{2}\right) / 2 h(R)$. Replacing $R^{2}$ by $k R$ in the second equality in (2.3) we see that $k h(R) R=2 k h(R) R$. Using that $h(R) R \neq 0$, it follows that $k=0$, hence (2.4) implies that $R^{2}=0$ as desired. This proves (2.2). Multiplying (2.1) by $R$ we see that $R a R=0$ for any $a$ in $\mathfrak{A}$. Taking $a=R^{*}$ it follows that $R R^{*} R=0$; multiplying on the left by $R$ we see that $\left(R^{*} R\right)^{2}=0$, hence that $R=0$. This contradiction completes the proof of Proposition 2.1.

In the proof of Proposition 2.1 we do not really need that $\mathfrak{A}$ is a $C^{*}$-algebra; all we need is that $\mathfrak{A}$ is a topological algebra with the property that the only element $R$ in $\mathfrak{A}$ satisfying $R a R=0$ for all $a$ in $\mathfrak{A}$ is $R=0$.

Theorem 2.2. Let $\delta$ be a densely defined closable $*$-derivation of a $C^{*}$-algebra. If the rank of $\delta$ is at most one, then $\delta=0$.

Proof. Suppose $\mathfrak{A}$ is a non-degenerate $C^{*}$-algebra of operators on some Hilbert space $\mathcal{H}$, and that $\delta$ is a rank one derivation densely defined closed $*$-derivation on $\mathfrak{A}$. Let $h, R$ and $b$ be as in the proof of Proposition 2.1. Then

$$
h\left(b^{*}\right) R=\delta\left(b^{*}\right)=\delta(b)^{*}=R^{*} .
$$

In particular $h\left(b^{*}\right)$ has absolute value one. Let $k$ be a square root of $\overline{h\left(b^{*}\right)}$, and let

$$
h^{\prime}(a)=k h(a) \text { and } R^{\prime}=\bar{k} R .
$$

Then $\delta(a)=h^{\prime}(a) R^{\prime}$ for all $a$ in $\mathfrak{A}$ and

$$
R^{\prime *}=k R^{*}=k h\left(b^{*}\right) R=\bar{k} R=R^{\prime} .
$$

So we may assume

$$
R^{*}=R .
$$

From the proof of (2.1) above we see that

$$
R a+a R=\lambda(a) R
$$

for all $a$ in $\mathfrak{A}$, where $\lambda$ is a continuous linear functional on $\mathfrak{A}$ and $\lambda\left(a^{*}\right)=\overline{\lambda(a)}$ for all $a$ in $\mathfrak{A}$. From (2.8) we see that both the range and kernel of $R$ are invariant under any $a$ in $\mathfrak{A}$. Let $\mathcal{H}_{1}$ denote the closure of the range of $R$ and let $\mathcal{H}_{0}$ denote the kernel of $R$. The $\mathfrak{A}$ is block diagonal with respect to the decomposition $\mathcal{H}=\mathcal{H}_{1} \oplus \mathcal{H}_{0}$ of the underlying Hilbert space. Applying (2.8) with $a=R$ we get

$$
R^{2}=\frac{\lambda(R)}{2} R .
$$

Using (2.7) and the definition of $\mathcal{H}_{1}$ and of $\mathcal{H}_{0}$ we see that $R=R_{1} \oplus 0$, where $R_{1}^{*}=R_{1}$. In particular (2.9) tells us that $R_{1}^{2}=\lambda(R) R_{1} / 2$; it follows that $R_{1}=$ $(\lambda(R) / 2) \mathbf{1}_{1}$, where $\mathbf{1}_{1}$ denotes the identity operator on $\mathcal{H}_{1}$. Thus replacing $\delta$ by $(2 / \lambda(R)) \delta$, if necessary, we may assume that $R=\mathbf{1}_{1} \oplus 0$. If $a$ in $\mathfrak{A}$ has block diagonal form $a=a_{1} \oplus a_{0}$, then it follows from (2.8) that $2 a_{1}=\lambda(a) \mathbf{1}_{1}$. In particular each 
$a$ in $\mathfrak{A}$ is of the form $\left(k \mathbf{1}_{1}\right) \oplus a_{0}$, where $k$ is a complex number and $a_{0}$ is a bounded linear operator on $\mathcal{H}_{0}$. Let $\mathfrak{A}_{0}$ be the subalgebra of $\mathfrak{A}$ consisting of the elements of the form $a=0 \oplus a_{0}$.

By adjoining $\mathbf{1}=\mathbf{1}_{1} \oplus \mathbf{1}_{0}$ to $\mathfrak{A}$ and extending $\delta$ in the natural way we may assume that 1 is in the domain $\mathcal{D}(\delta)$ of $\delta$, see e.g. [BR76] or [BR79]. Let $\delta_{0}$ denote the linear map on $\mathfrak{A}_{0}$ determined by $\delta_{0}(a)=\left(0 \oplus \mathbf{1}_{0}\right) \delta(a)$ for each $a$ in $\mathcal{D}\left(\delta_{0}\right)=\mathcal{D}(\delta) \cap \mathfrak{A}_{0}$. Then $\delta_{0}$ is a densely defined closed derivation on $\mathfrak{A}_{0}$. It follows that $0 \oplus \mathbf{1}_{0}$ is in $\mathcal{D}\left(\delta_{0}\right)$. But then $R=\mathbf{1}-\left(0 \oplus \mathbf{1}_{0}\right)$ is in $\mathcal{D}(\delta)$. We can now apply Proposition 2.1 to complete the proof.

Example 2.3. Let $\mathfrak{A}$ be the algebra of upper $3 \times 3$ matrices. Then the derivation $\delta(a)=b a-a b$ will have rank one (or two, or three) for a suitable choice of $b$ in $\mathfrak{A}$. It follows that any positive integer is a candidate for the dimension of the range of some derivation of a (suitable finite dimensional) nest algebra. It is, however, not clear what happens in a general nest, e.g., can the nest algebra of a continuous nest have rank one derivations?

Preliminary calculations suggest that the range of a $*$-derivation can only have even dimension. However, this is not needed below.

\section{Derivations anticommuting With a group aCtion}

In this section we consider a derivation $\delta$ of a $C^{*}$-algebra $\mathfrak{A}$ with identity $\mathbf{1}$ and an action $\alpha$ of a group $G$ on $\mathfrak{A}$. We show (given some mild technical condition) that if $\alpha$ acts ergodically, and $\alpha$ and $\delta$ anticommute, then $\delta=0$. That $\alpha$ acts ergodically means that the fixed point algebra

$$
A^{\alpha}=\left\{a \in A: \alpha_{g}(a)=a, \text { for all } g \in G\right\}
$$

equals $\mathbb{C} \mathbf{1}$, the scalar multiples of $\mathbf{1}$. Recall, $\alpha$ and $\delta$ anticommute provided

$$
\alpha_{g} \delta \subset \delta \alpha_{g^{-1}}
$$

for all $g$ in $G$. The situation where $G$ is a $d$-dimensional torus and $\alpha$ and $\delta$ commute, i.e.,

$$
\alpha_{g} \delta \subset \delta \alpha_{g}
$$

for all $g$ in $G$, and $\delta$ is defined on the $\alpha$-smooth elements in $\mathfrak{A}$, was shown to have far reaching consequences for the structure of $\delta$ in [BEJ84], where $\alpha$ is an ergodic (faithful) action. (See [Br86] and [Sa91] for some further developments.) The basis for [BEJ84] was the observation [St74] that there exist unitaries $U_{\gamma}$ in $\mathfrak{A}$ such that $\alpha_{g}\left(U_{\gamma}\right)=(g, \gamma) U_{\gamma}$ for all $g$ in $G$ and all $\gamma$ in the dual group $\widehat{G}$. Here $(g, \gamma)$ denotes the pairing between $G$ and $\widehat{G}$. It then follows from $(3.2)$ that $\delta\left(U_{\gamma}\right)=\beta(\gamma) U_{\gamma}$ for all $\gamma$ in $\widehat{G}$, where $\beta$ is a scalar valued function defined on the dual group $\widehat{G}$. If one assumes (3.1) in place of (3.2), then the corresponding result is $\delta\left(U_{\gamma}\right)=\beta(\gamma) U_{\gamma^{-1}}$ for all $\gamma$ in $\widehat{G}$. The result proved below does not use this, but it implies $\beta \equiv 0$.

Theorem 3.1. Let $\mathfrak{A}$ be a $C^{*}$-algebra with identity $\mathbf{1}, \delta$ a closable densely defined *-derivation on $\mathfrak{A}, G$ a compact group with $\left\{g^{2}: g \in G\right\}$ dense in $G$, and let $\alpha$ be an ergodic action of $G$ on $\mathfrak{A}$. If $\alpha$ and $\delta$ anticommute, then $\delta=0$. 
Proof. By replacing $\delta$ by its closure, if necessary, we may assume that $\mathbf{1}$ is in the domain $\mathcal{D}(\delta)$ of $\delta$. Suppose $\delta$ has rank greater than one. Let $a$ and $b$ be in $\mathcal{D}(\delta)$; then

$$
\alpha_{g} \delta(a b)=\left(\alpha_{g} \delta(a)\right) \alpha_{g^{-1}}(b)+\alpha_{g^{-1}}(a) \alpha_{g} \delta(b)
$$

for all $g$ in $G$. Hence, density of $\left\{g^{2}: g \in G\right\}$ in $G$ implies

$$
\delta(a b)=\delta(a) \alpha_{g}(b)+\alpha_{g}(a) \delta(b)
$$

for all $g$ in $G$. Now, if $a$ is in $\mathfrak{A}$, then

$$
P_{0} a=\int_{G} \alpha_{g}(a) d g
$$

determines a projection of $\mathfrak{A}$ onto the fixed point algebra $\mathfrak{A}^{\alpha}=\mathbb{C} 1$. Hence, integrating (3.3) yields the existence of two scalar valued functions $\phi$ and $\psi$ defined on $\mathcal{D}(\delta)$, such that

$$
\delta(a b)=\phi(b) \delta(a)+\psi(a) \delta(b) .
$$

Expanding $\delta\left(\left(k a_{1}+a_{2}\right) b\right)=k \delta\left(a_{1} b\right)+\delta\left(a_{2} b\right)$ using (3.4), where $k$ is a scalar and $a_{1}$, $a_{2}$ and $b$ are in the domain of $\delta$, it follows that $\psi$ is a linear functional. Similarly, one can show that $\phi$ is a linear functional. Expanding both sides of $\delta((a b) c)=\delta(a(b c))$ using Leibnitz's rule and (3.4) we get

$$
\begin{aligned}
& \phi(b) \phi(c) \delta(a)+\phi(c) \psi(a) \delta(b)+\psi(a b) \delta(c) \\
= & \phi(b c) \delta(a)+\phi(c) \psi(a) \delta(b)+\psi(a) \psi(b) \delta(c)
\end{aligned}
$$

for all $a, b$ and $c$ in $\mathcal{D}(\delta)$. Temporarily, fix $b$ and $c$ in $\mathcal{D}(\delta)$. If $\delta(c)=0$, pick $a$ in $\mathcal{D}(\delta)$ so that $\delta(a) \neq 0$; it follows that $\phi(b) \phi(c)=\phi(b c)$. If $\delta(c) \neq 0$, pick $a$ in $\mathcal{D}(\delta)$ so that $\delta(a)$ and $\delta(c)$ are linearly independent; then again $\phi(b) \phi(c)=\phi(b c)$. A similar argument can be applied to $\psi$. We have shown

$$
\phi(a) \phi(b)=\phi(a b) \text { and } \psi(a) \psi(b)=\psi(a b)
$$

for all $g$ in $G$ and all $a$ and $b$ in $\mathcal{D}(\delta)$. Using (3.4) with either $a=\mathbf{1}$ or $b=\mathbf{1}$ it follows that $\phi(\mathbf{1})=\psi(\mathbf{1})=1$. Now a simple calculation using (3.1) and (3.4) shows that

$$
\phi(b) \delta(a)+\psi(a) \delta(b)=\phi\left(\alpha_{g} b\right) \delta(a)+\psi\left(\alpha_{g} a\right) \delta(b)
$$

for all $g$ in $G$ and all $a$ and $b$ in $\mathcal{D}(\delta)$. It follows that $\phi(b)=\phi\left(\alpha_{g} b\right)$ and $\psi(a)=$ $\psi\left(\alpha_{g} a\right)$ for all $g$ in $G$ and all $a$ and $b$ in $\mathcal{D}(\delta)$. Expanding $\delta\left(a P_{0} b\right)$ in two ways, first using (3.4) and then using Leibnitz's rule we get

$$
\phi\left(P_{0} b\right) \delta(a)+\psi(a) \delta\left(P_{0} b\right)=\delta(a) P_{0} b+a \delta\left(P_{0} b\right)
$$

for all $a$ and $b$ in $\mathcal{D}(\delta)$. But $\delta\left(P_{0} b\right)=0$, since $P_{0} b$ is in $\mathfrak{A}^{\alpha}=\mathbb{C} \mathbf{1}$, so it follows that $\phi\left(P_{0} b\right) \mathbf{1}=P_{0} b$ for all $b$ in $\mathcal{D}(\delta)$. Hence, (3.5) implies $\left(P_{0} a\right)\left(P_{0} b\right)=\left(P_{0} a b\right)$ for all $a$ and $b$ in $\mathfrak{A}$. It follows that $\mathfrak{A}=\mathbb{C} 1$, contradicting that $\delta$ has rank $\geqslant 2$. So $\delta$ has rank $\leqslant 1$; now an application of Theorem 2.2 completes the proof.

\section{ACKNOWLEDGMENT}

The author would like to thank Anthony B. Evans, Costel Peligrad and Derek Westwood for useful conversations. In particular Peligrad and Westwood proved special cases of Proposition 2.1, and Evans supplied an example similar to the example above (but not in a nest algebra). 


\section{REFERENCES}

[Br86] O. Bratteli, Dissipations and Group Actions on $C^{*}$-algebras, Springer Lecture Notes in Mathematics, Vol. 1229, Springer-Verlag, Berlin, 1986. MR 88e:46050

[BEJ84] O. Bratteli, G. A. Elliott and P. E. T. Jorgensen, Decomposition of unbounded derivations into invariant and approximately inner parts, J. Reine Angew. Math. 346(1984), 166193. MR 85j:46106

[BR76] O. Bratteli and D. W. Robinson, Unbounded derivations of $C^{*}$-algebras II, Commun. Math. Phys. 46(1976), 11-30. MR 52:11608

[BR79] O. Bratteli and D. W. Robinson, Operator Algebras and Quantum Statistical Mechanics I, Texts and Monographs in Physics, Springer-Verlag, New York-Heidelberg-Berlin, 1979. MR 81a:46070

[JM84] P. E. T. Jorgensen ad R. T Moore, Operator Commutation Relations, Mathematics and Its Applications, D. Reidel Publishing Company, Dordrecht-Boston-Lancaster, 1984.

[Sa91] S. Sakai, Operator Algebras in Dynamical Systems, Encyclopedia of Mathematics and its Applications 41, Cambridge University Press, Cambridge, 1991. MR 92h:46099

[St74] E. Størmer, Spectra of ergodic transformations, J. Funct. Anal. 15(1974), 202-215. MR 51:13715

Department of Mathematics, Wright State University, Dayton, Ohio 45435

E-mail address: steen@math.wright.edu 